\title{
BLOCK IDEMPOTENTS OF TWISTED GROUP ALGEBRAS ${ }^{1}$
}

\section{W. F. REYNOLDS}

In [4] Conlon has successfully generalized much of the theory of modular representations to the projective case. However his generalization [4, p. 166] of one of Brauer's main theorems on blocks [3, 10B], [5] is not entirely satisfactory. In Theorem 1 we present another generalization which is closer than Conlon's to the original Brauer theorem, and in Theorem 2 we indicate an application involving the number of blocks with a given defect group.

Let $G$ be a finite group and $\Omega$ a field of prime characteristic $p$. A twisted group algebra $\Gamma(G)$ of $G$ over $\Omega$ is an associative $\Omega$-algebra with a basis consisting of elements $(g)$ in one-to-one correspondence with the elements $g$ of $G$, with multiplication determined by equations

$$
(g)(h)=\epsilon_{g, h}(g h), \quad g, h \in G,
$$

where $0 \neq \epsilon_{g, h} \in \Omega$. By associativity, $\boldsymbol{\epsilon}=\left\{\boldsymbol{\epsilon}_{g, h}\right\}$ must be a factor set of $G$ in $\Omega$. It is well known that the projective representations of $G$ in $\Omega$ with factor set $\epsilon$ can be identified with the representations of $\Gamma(G)$ [6].

For any $g \in G$, define

$$
C^{e}(g)=\left\{x \in G:(x)^{-1}(g)(x)=(g)\right\} .
$$

It is evident that $C^{e}(g)$ is a subgroup of the centralizer $C(g)$ of $g$ in $G$. Let us call $g$ e-regular provided that $C^{\epsilon}(g)=C(g)$. A short calculation shows that

$$
C^{e}\left(h^{-1} g h\right)=h^{-1} C^{e}(g) h, \quad g, h \in G ;
$$

hence the set of all $\epsilon$-regular elements is a union of conjugate classes of $G$, which we call the $\epsilon$-regular classes of $G$.

We assume ${ }^{2}$ that $\Gamma(G)$ satisfies the following conditions:

$$
\begin{aligned}
(h)^{-1}(g)(h) & =\left(h^{-1} g h\right), & g, h & \in G, g \text { e-regular; } \\
\left(g^{-1}\right) & =(g)^{-1}, & g & \in G .
\end{aligned}
$$

(Condition (2) is never an essential restriction; and neither is (3) if $\Omega$ is algebraically closed $[4, \S 1]$.)

Received by the editors February 15, 1965.

1 This work has been supported in part by the National Science Foundation, through Harvard University, under Grant NSF-G-23833.

${ }^{2}$ In fact we do not need to assume (3), since it is not required in the proof of Conlon's theorem. 
For each $\epsilon$-regular class $K$, let $(K)=\sum_{g \in K}(g)$; these $\epsilon$-regular class sums $(K)$ form a basis of the center $\Lambda(G)$ of $\Gamma(G)$. As usual, we call any $p$-Sylow subgroup of $C(g)$ for any $g \in K$ a defect group of $K$. For any block idempotent, i.e. primitive idempotent, $e$ of $\Lambda(G)$, write $e=\sum_{K} f_{K}(K), f_{K} \in \Omega$. Then the largest of the defect groups of the $K$ for which $f_{K} \neq 0$ can be called a defect group of $e$; this is uniquely determined up to conjugacy in $G[4, \S 3]$.

Let $D$ be an arbitrary $p$-subgroup of $G$. Let $C(D)$ be the centralizer of $D$ in $G$, and denote the normalizer $N(D)$ of $D$ in $G$ by $H$. For each $\epsilon$-regular class $K$, set

$$
s((K))=\sum_{g \in K \cap C(D)}(g) .
$$

By $[4, \S 3]$, extending $s$ by linearity gives an $\Omega$-algebra homomorphism $s: \Lambda(G) \rightarrow \Lambda(H)$, where $\Lambda(H)$ is the center of the twisted group algebra $\Gamma(H)$ of $H$ whose factor set is the restriction $\epsilon \mid H$ of $\epsilon$ to $H$. Adapting our terminology to $H$ in the obvious way, we can now state:

THEOREM 1. The homomorphism $s$ determines a one-to-one correspondence $e \leftrightarrow s(e)$ between the block idempotents of $\Lambda(G)$ which have $D$ as one of their defect groups and the block idempotents of $\Lambda(H)$ which have $D$ as their unique defect group.

We shall show that Theorem 1 follows from Conlon's theorem. The lgtter states that $e \leftrightarrow s(e)$ is a one-to-one correspondence between the block idempotents of $\Lambda(G)$ which have $D$ as one of their defect groups and the primitive idempotents of $U(D)$, where $U(D)$ is a subalgebra of $\Lambda(H)$ which has as a basis those $(\epsilon \mid H)$-regular class sums $(L)$ of $H$ such that $L$ has defect group $D$ and consists of $\epsilon$-regular elements. (Since only $\epsilon$-regular elements are involved, these class sums are defined in $\Lambda(H)$, even though the analogue of (2) for $\epsilon \mid H$ need not hold.) Furthermore each primitive idempotent of $U(D)$ is a sum of block idempotents of $\Lambda(H)$ which have defect group $D$.

As Conlon points out, the complication in his theorem is due to the fact that an $(\epsilon \mid H)$-regular element need not be $\epsilon$-regular. However, we can prove:

Lemma. Every $(\epsilon \mid H)$-regular element whose conjugate class in $H$ has defect group $D$ is $\epsilon$-regular.

This lemma implies that the $(\epsilon \mid H)$-regular class sums $(L)$ of $H$ such that $L$ has defect group $D$ form a basis of $U(D)$, and hence that $U(D)$ contains all block idempotents of $\Lambda(H)$ with defect group $D$. Therefore these idempotents of $\Lambda(H)$ are precisely all the primitive 
idempotents of $U(H)$. This proves that Theorem 1 follows from Conlon's theorem.

It remains to prove the lemma. Let $h \in H$ satisfy the hypothesis of the lemma. Since $h$ is $(\epsilon \mid H)$-regular, $C(h) \cap H \subseteq C^{\epsilon}(h)$. Since $D$ is the unique defect group of the class of $h$ in $H, D$ is a $p$-Sylow subgroup of $C(h) \cap H$. Then the second paragraph of the proof of [5, Lemma 3.4] shows that $D$ is a $p$-Sylow subgroup of $C(h)$, and hence also of $C^{\epsilon}(h)$. For any $x \in C(h), x^{-1} D x$ is a $p$-Sylow subgroup of $x^{-1} C^{\epsilon}(h) x$, which equals $C^{e}(h)$ by (1). Then $x^{-1} D x=y^{-1} D y$ for some $y \in C^{\epsilon}(h)$, and $x y^{-1} \in N(D) \cap C(x)=H \cap C(x) \subseteq C^{\epsilon}(h)$. Hence $x \in C^{\epsilon}(h)$, so that $h$ is $\epsilon$-regular as required.

Theorem 1 can be applied in conjunction with the methods of Bovdi [1] to generalize [1, Theorems 1 and 2] as follows (cf. [2, Corollary 1]).

THEOREM 2. The number of block idempotents of $\Lambda(G)$ with $D$ as a defect group is less than or equal to the number of p-regular $\epsilon$-regular classes $K$ of $G$ with $D$ as a defect group such that $(K)$ is not a nilpotent element of $\Lambda(G)$.

Equality holds here if $G$ has a normal subgroup $T$ of p-power index such that $T$ has a normal $p$-Sylow subgroup, while $\Omega$ is algebraically closed.

In a later paper we shall give a proof of a more general form of Theorem 2.

I wish to thank Dr. Conlon for some helpful correspondence.

\section{REFERENCES}

1. A. A. Bovdi, The number of blocks of characters of a finite group with a given defect, Ukrain. Mat. Ž. 13 (1961), 136-141. (Russian)

2. R. Brauer, On the arithmetic in a group ring, Proc. Nat. Acad. Sci. U.S.A. 30 (1944), 109-114.

3. —_ Zur Darstellungstheorie der Gruppen endlicher Ordnung I, Math. Z. 63 (1956), 406-444.

4. S. B. Conlon, Twisted group algebras and their representations, J. Austral. Math. Soc. 4 (1964), 152-173.

5. A. Rosenberg, Blocks and centres of group algebras, Math. Z. 76 (1961), 209-216.

6. M. Tazawa, Über die Darstellung der endlichen verallgemeinerten Gruppen, Sci. Rep. Tôhoku Univ. Ser. I 23 (1934), 76-88.

TUFTS UNIVERSITY 